\title{
Experimental Demonstration and Theoretical Analysis of Slow Light in a Semiconductor Waveguide at GHz Frequencies
}

Mørk, Jesper; Kjær, Rasmus; Poel, Mike van der; Oxenløwe, Leif Katsuo; Yvind, Kresten

Published in:

Conference on Lasers and Electro-Optics, 2005. (CLEO).

Publication date:

2005

Document Version

Publisher's PDF, also known as Version of record

Link back to DTU Orbit

Citation (APA):

Mørk, J., Kjær, R., Poel, M. V. D., Oxenløwe, L. K., \& Yvind, K. (2005). Experimental Demonstration and Theoretical Analysis of Slow Light in a Semiconductor Waveguide at GHz Frequencies. In Conference on Lasers and Electro-Optics, 2005. (CLEO). (Vol. 1). IEEE.

\section{General rights}

Copyright and moral rights for the publications made accessible in the public portal are retained by the authors and/or other copyright owners and it is a condition of accessing publications that users recognise and abide by the legal requirements associated with these rights.

- Users may download and print one copy of any publication from the public portal for the purpose of private study or research.

- You may not further distribute the material or use it for any profit-making activity or commercial gain

- You may freely distribute the URL identifying the publication in the public portal 


\title{
Experimental Demonstration and Theoretical Analysis of Slow Light in a Semiconductor Waveguide at GHz Frequencies
}

\author{
Jesper Mørk, Rasmus Kjær, Mike van der Poel, Leif Oxenløwe, and Kresten Yvind \\ Research Center COM, Technical University of Denmark, Build. 345v, DK-2800 Kgs. Lyngby, Denmark \\ Tel: +45 45255765, Fax: +4545936581, E-mail: jm@com.dtu.dk \\ Abstract: We demonstrate slow-down of light by a factor of two in a semiconductor waveguide at room \\ temperature with a bandwidth of $16.7 \mathrm{GHz}$. The achievable delay is shown to be limited by the short lifetime. \\ (C) 2005 Optical Society of America \\ OCIS codes: (190.5970) Semiconductor nonlinear optics, (230.1150) All-optical devices
}

\section{Introduction}

The demonstration of slow light propagation in ultracold atomic gasses [1] has spurred significant interest in this field, due both to the fundamental aspects as well as the possibility to implement all-optical buffers [2]. Recently, slow-light propagation was demonstrated in a ruby crystal at room temperature [3] as well as in a quantum well at low temperature [4]. These latter demonstrations used the effect of coherent population oscillations (CPO), which may be viewed as a wave-mixing interaction where the effective group velocity of a probe field is affected by a strong pump field. Importantly, in CPO, the dispersion profile of the spectral hole seen by the probe field is governed by the population lifetime rather than the dephasing time [3], leading to relaxed requirements for the conditions under which light slow-down may be observed. For practical applications, semiconductor waveguides are attractive and we present, for the first time we believe, the experimental demonstration of slow-down in a short, 100 $\mu \mathrm{m}$, waveguide at room temperature and at high frequencies. Our theoretical analysis, however, also indicates some fundamental issues that must be considered in the application of this effect, e.g., to store a random bit-sequence.

\section{Experimental set-up and results}

We use the experimental technique of [3] to measure the changes in propagation time through a sample, see Fig. 1. In short, a weak (double-sideband) probe field is generated by applying a small sinusoidal amplitude modulation on a CW laser beam, which is transmitted through the device. The modulation frequency determines the pump-probe detuning [3]. By measuring the phase delay of the output signal relative to the input, the time delay can be easily inferred. The measurement of small time delays requires a careful calibration of the optical response of the system. The device used here is a commercial p-i-n electro-absorption modulator with a length of approximately $100 \mu \mathrm{m}$, the absorption of which is controlled by a reverse voltage.

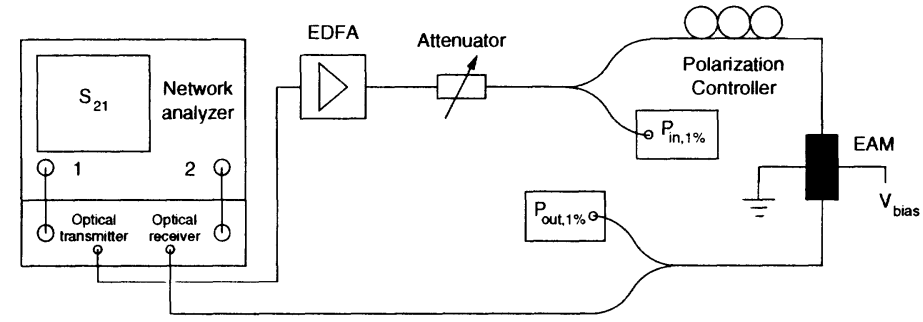

Fig. 1. Experimental set-up for measuring the propagation speed of a modulated optical signal in a semiconductor waveguide device.

Fig. 2 shows the measured intensity transmission of the signal and the phase (and time) delay versus applied reverse voltage for different input pump power levels at a detuning frequency of $16.7 \mathrm{GHz}$. As the pump power is increased, the absorption saturates and the time delay increases. The optical control of the delay time is the basis for suggestions to use this effect to implement an all-optical buffer [4], but the experimental results presented here also demonstrate the possibility for simple electrical control. The reversal of the intensity dependence of the transmission beyond $2.5 \mathrm{~V}$ is due to temperature effects induced by the photo-current [5] and plays no importance at the high modulation frequency considered here. The maximum slow-down observed is approximately $2.1 \mathrm{ps,} \mathrm{corresponding}$ to a near doubling of the propagation time through the device or a change of the group refractive index $\Delta n_{g} \approx 3$. This absolute value of the time delay may appear modest but, as will be made clear below, this value should be compared 


\section{CMCC5}

to the lifetime itself. At $2 \mathrm{~V}$ reverse bias the lifetime is estimated to be as short as $10 \mathrm{ps}$, reduced by carrier sweepout from the active region. We thus achieve a time delay of approximately $20 \%$ of the lifetime. In [3], a time delay as large as $1.26 \mathrm{~ms}$ was achieved, corresponding to $28 \%$ of the $4.45 \mathrm{~ms}$ lifetime in that experiment. However, a long lifetime also limits the modulation frequency, which was on the order of tens of $\mathrm{Hz}$ in [3].
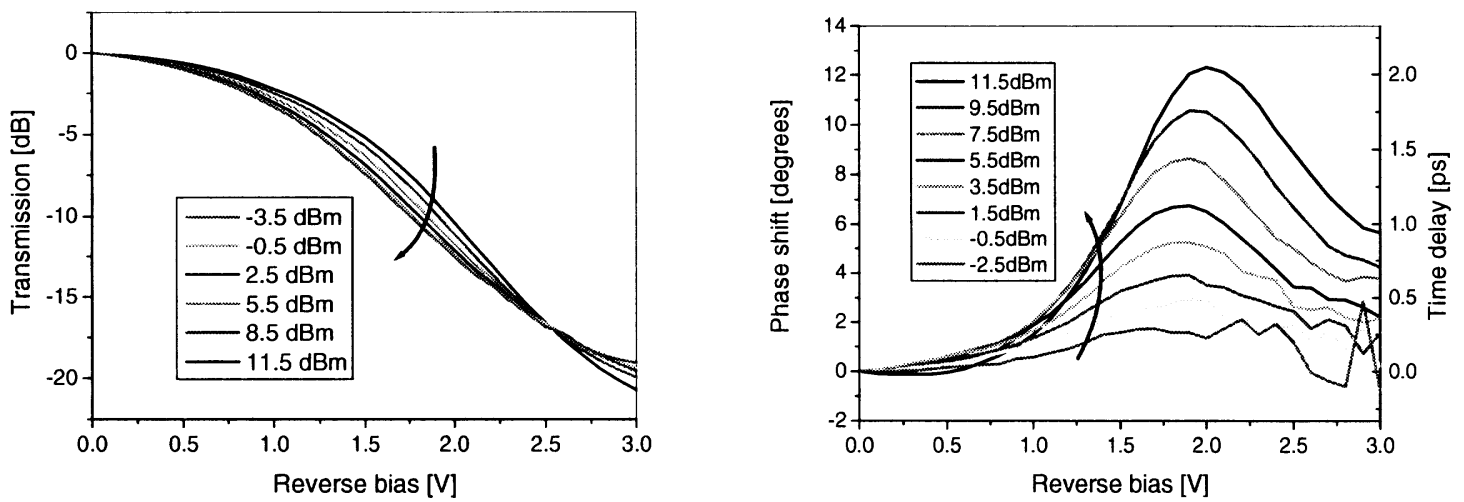

Fig. 2. Left: Measured transmission versus voltage for different input power levels. Right: Phase shift and time delay (right axis) versus reverse bias for different input power levels. Frequency detuning: $16.7 \mathrm{GHz}$.

\section{Modelling results}

The pump-induced changes of the probe may be analyzed by using models for four-wave mixing in active semiconductor waveguides [6]. At detuning frequencies ranging up to about $1 \mathrm{THz}$, the dominant mechanism mediating wave interactions is pulsations of the carrier density in the active region; the corresponding susceptibility for the probe has a dispersion governed by the lifetime of the carrier density, an example may be found in [6]. Using this approach we can calculate the group index seen by the probe and get the total time delay by integrating over the device length, taking into account the absorption (and thus index) saturation induced by the pump. Alternatively, one can make a small-signal analysis for the complex modulation amplitude of the propagating signal.
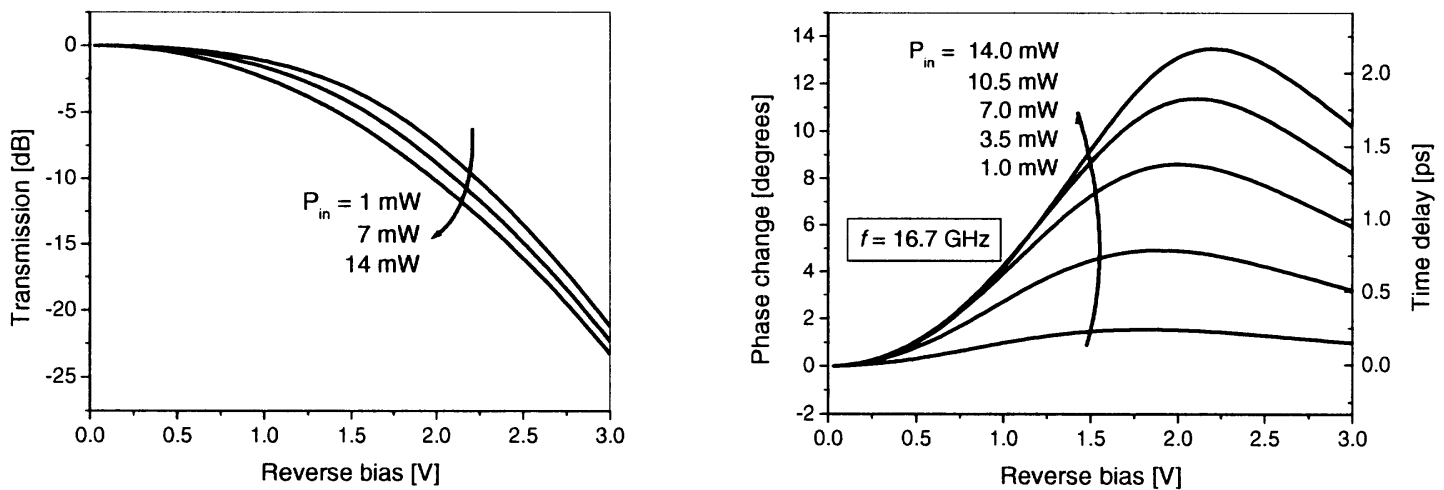

Fig. 3. Left: Calculated transmission versus voltage for different input power levels. Right: Calculated phase shift and time delay versus reverse bias for different input power levels. Frequency detuning: $16.7 \mathrm{GHz}$.

Fig. 3 shows calculated results corresponding to the measurements of Fig. 2. The carrier lifetime reduces (exponentially) with the reverse voltage and has a value of $10 \mathrm{ps}$ at $2 \mathrm{~V}$. The corresponding saturation power; inversely proportional to the lifetime and the differential absorption, attains a value of $11 \mathrm{~mW}$ at $2 \mathrm{~V}$. The model is seen to account well for the experimental observations. The appearance of a local maximum for the time delay at a specific voltage can be traced back to the voltage dependence of the lifetime. Temperature effects are not included in the model, and the calculated transmission therefore fails to show the measured saturation reversal beyond $2.5 \mathrm{~V}$ [5]. 


\section{CMCC5}

\section{Discussion}

Experimentally, we were limited in the range of absorption and input power levels that could be investigated. Fig. 4 shows modeling results for a wider range of values; the saturated transmission (power out relative to power in) and time delay are plotted versus input power normalized by the saturation power, $S / S_{\text {sat }}$. The different curves correspond to different levels of absorption, indicated by the low-power transmission. $T_{0}$. The time delay is here given relative to the carrier lifetime, i.e., $\xi=\Delta t / \tau$. The calculations were carried out in the limit of zero detuning frequency, where the absolute time delay is largest [3]. First, we notice that the theory predicts a local maximum for the delay in dependence on the power level, which occurs because of absorption saturation and power broadening of the induced dispersion. We also see that $\xi<1$, i.e., the time delay remains smaller than the carrier lifetime. Our model shows that this is a general result for the mechanism of carrier density pulsations considered. This limitation can also be qualitatively understood, considering that the delay arises from the saturable absorption property of the medium.
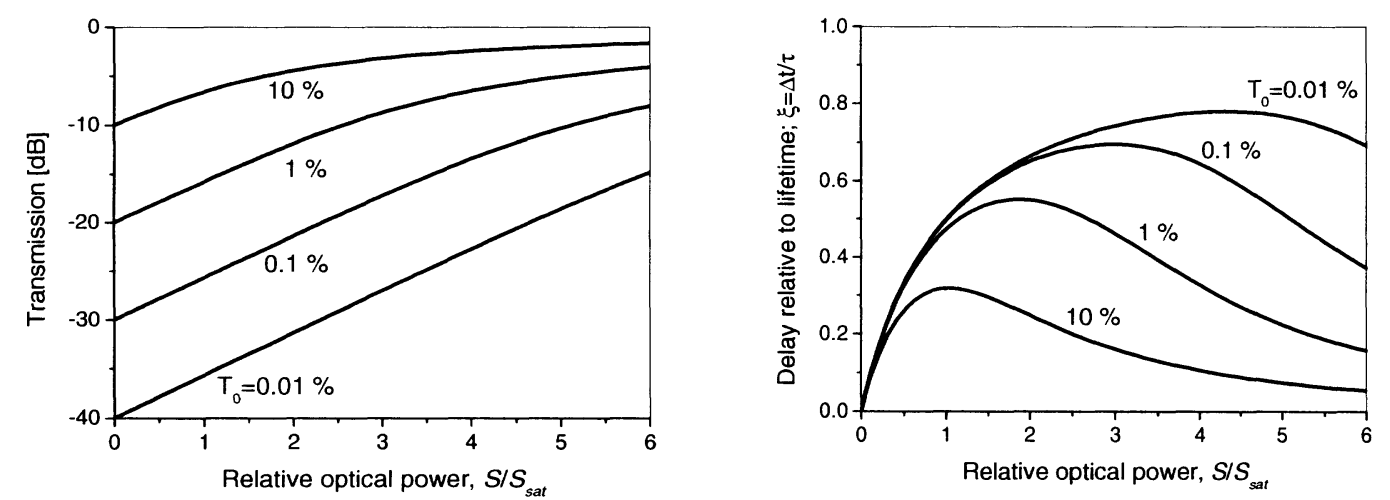

Fig. 4. Calculated transmission (left) and relative time delay $\xi=\Delta t / \tau$ (right) versus optical power relative to saturation power for different levels of (small-signal) transmission $T_{0}$ through the device.

\section{Conclusions}

We have experimentally demonstrated the slow-down of light in a semiconductor waveguide at room temperature and at frequencies beyond $15 \mathrm{GHz}$ using the effect of coherent pulsations of the carrier density. The maximum time delay observed reflects an approximately two-fold increase of the group refractive index, corresponding to a time delay of approximately $20 \%$ of the carrier (population) lifetime. The experimental observations are well-explained by a model accounting for the absorption saturation in the waveguide, when using a lifetime that depends on the reverse bias. The model further predicts that the obtainable time delay is limited by the carrier lifetime, which seems to be in agreement with other reported experiments using the effect of coherent population oscillations [3].

Since the achievement of light slow-down requires a relatively strong saturation of the medium, the bit-period of an optical signal needs to be comparable to or longer than the lifetime in order to avoid patterning effects. With the time delay being limited by the carrier lifetime, this suggests that a straightforward application of the mechanism of coherent population oscillations to store a bit-stream would be limited to less than one bit.

\section{References}

[1] L.V. Hau. S.E. Harris. Z. Dutton, and C.H. Behroozi. "Light speed reduction to 17 meters per second un an ultracold atomic gas", Nature 397. $594-598(1999)$

[2] C.J. Chang-Hasnain. P.-C. Ku. J. Kim. S.-L. Chuang. "Variable optical buffer using slow light in semiconductor nanostructures", Proc. IEEE 91. $1884-1897(2003)$

[3] M.S. Bigelow, N.N. Lepeshkin. and R. Boyd. "Observation of ultraslow light propagation in a ruby crystal at room temperature". Phys. Rev. Lett. $90.113903-1-4(2003)$

[4] P.-C. Ku. F. Sedgwick, C.J. Chang-Hasnain. P. Palinginis, T. Li, H. Wang, S.-W. Chang, and S.-L. Chuang, "Slow light in semiconductor quantum wells". Opt. Lett. 19, 2291-2293 (2004)

[5] J. Shim. Member. B. Liu. and J. E. Bowers. "Dependence of transmission curves on input optical power in an electroabsorption modulator", IEEE J. Quantum Electron., 40. 1622-1628 (2004)

[6] A. Uskov, J. Mørk and J. Mark. "Wave mixing in semiconductor laser amplifiers due to carrier heating and spectral-hole burning”, IEEE J. Quantum Electron.. vol. 30, pp. 1769- 1781 (1994). 